Int. J. Curr. Res. Med. Sci. (2017). 3(12): 13-21

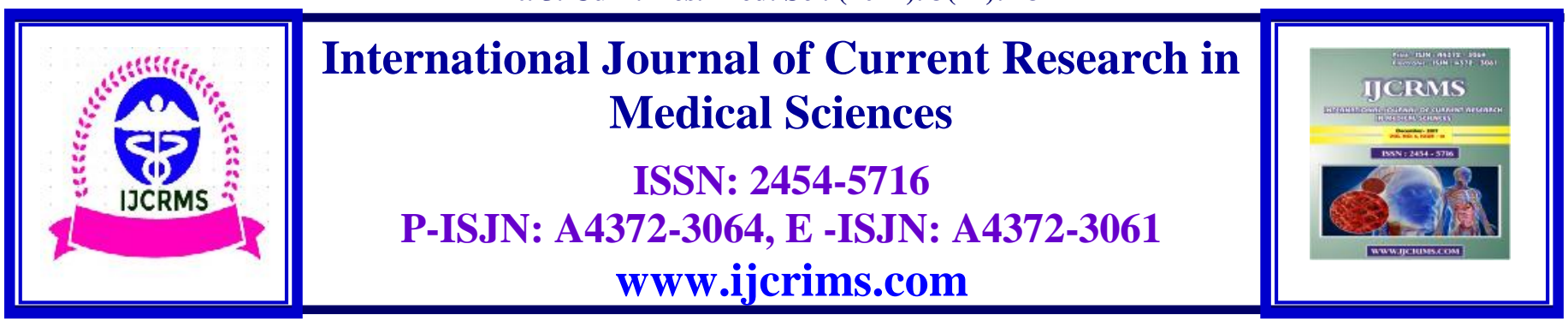

Review Article

Volume 3, Issue 12 -2017

DOI: http://dx.doi.org/10.22192/ijcrms.2017.03.12.003

\title{
Increasing Incidence of Brain Tumors
}

\author{
Dr. Anil Batta \\ Assoc. Professor, Department of Medical Biochemistry \\ Govt. Medical College, Amritsar. \\ E-mail:akbattafarid@yahoo.co.in
}

\begin{abstract}
A brain tumor is a collection of abnormal cells in the brain. Brain tumors can be either malignant (cancerous) or benign (unlikely to spread). However, there is a limited amount of space in the skull. Therefore, any brain tumoreven one that is benign - can interfere with the functions of your brain and body. Brain tumors can destroy brain cells, increase inflammation, and elevate the pressure in the brain. A primary brain tumor starts in your brain. When cancer cells from other parts of your body cause a tumor in your brain, it is called a "secondary" or "metastatic brain tumor." Secondary brain tumors are three times more common than primary brain tumors. All secondary brain tumors are malignant. Brain tumors are described by their location, tissue type, and the cells that make up the mass. The following types of brain tumors are commonly found in adults:

1. The following types of brain tumors are commonly found in adults: Gliomas come in different types. They range from non-cancerous to mixed cells. Glioblastomas are the most aggressive form of glioma.

2. Meningiomas are more common in adults in the 40-70 age group. They are typically non-cancerous. Meningiomas occur more commonly in women.

3. Schwannoma also occur in adults aged 40-70. They affect both men and women.

4. There are also other types of brain tumors that can be found in adults. Some rare types of adult brain tumors include pituitary tumors, primary lymphoma of the brain, and ependymomas.
\end{abstract}

Keywords: Tumors, Meningiomas, metastatic, Schwannoma 


\section{Introduction}
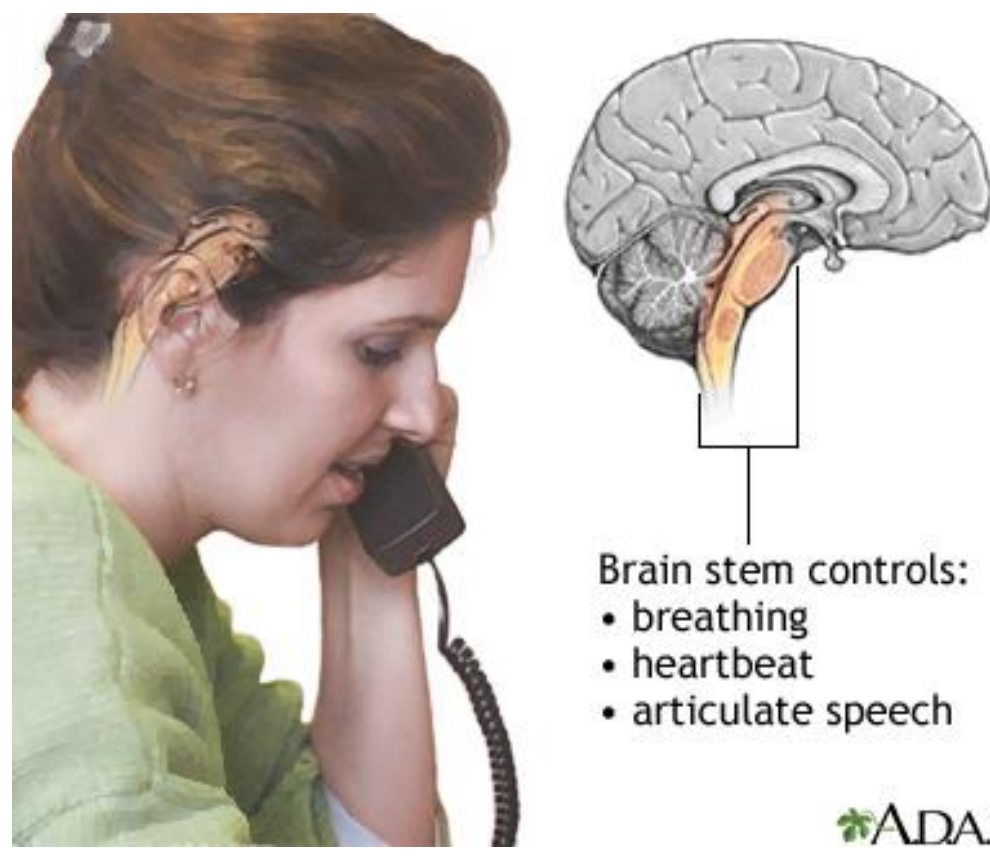

\%ADAM.

Doctors do not know what causes primary brain tumors. However, several risk factors have been identified.

\section{Introduction to brain tumor}

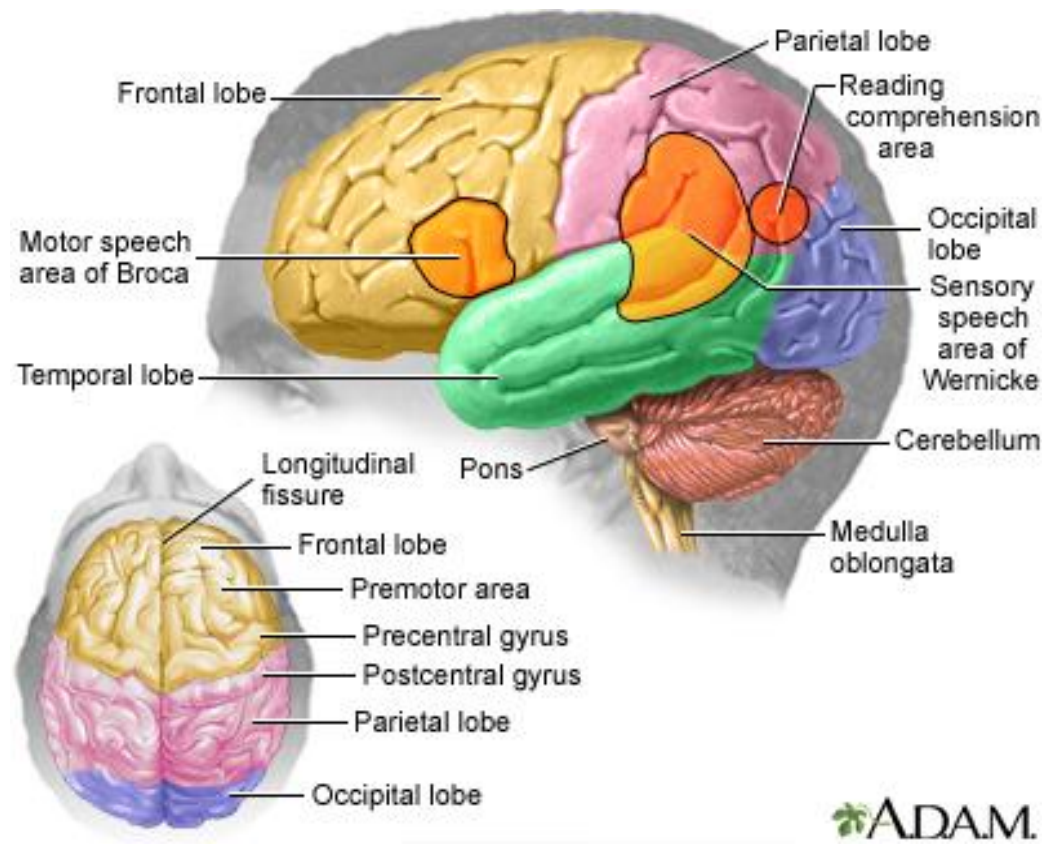

Some genetic diseases like Turcot syndrome (a condition characterized by abnormal cells and polyps) and neurofibromatosis (a tumor-causing genetic disorder).
Radiation treatments for brain cancer increase your risk of developing brain tumors.

There is no proof that cell phone use causes brain cancer. The National Cancer Institute reports that 
although cell phone use has increased significantly from 1992 to 2008 , the incidence of glioma has not seen a significant rise during that time period. However, researchers continue to study the question. It can take up to 10 years or longer for a brain tumor to manifest, requiring long-term studies.

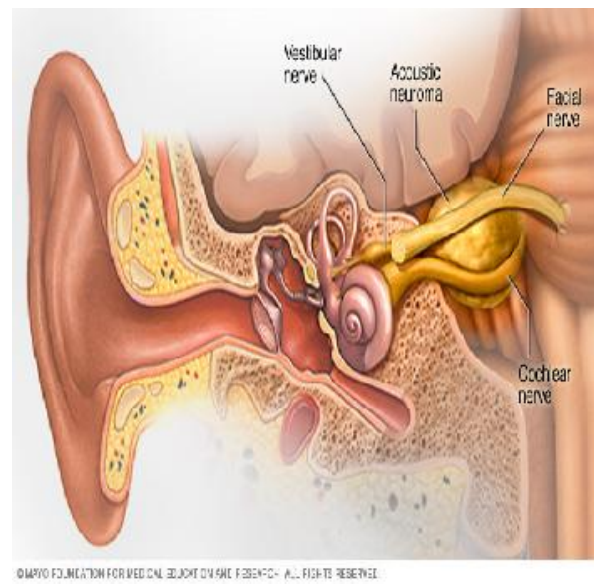

Acoustic neuroma (schwannoma)

\section{Brain tumors that begin in the brain}

Primary brain tumors originate in the brain itself or in tissues close to it, such as in the braincovering membranes (meninges), cranial nerves, pituitary gland or pineal gland. Primary brain tumors begin when normal cells acquire errors (mutations) in their DNA. These mutations allow cells to grow and divide at increased rates and to continue living when healthy cells would die. The result is a mass of abnormal cells, which forms a tumor.

\section{Cancer that begins elsewhere and spreads to the brain}

Secondary (metastatic) brain tumors are tumors that result from cancer that starts elsewhere in your body and then spreads (metastasizes) to your brain.

Secondary brain tumors most often occur in people who have a history of cancer. But in rare cases, a metastatic brain tumor may be the first sign of cancer that began elsewhere in your body. Secondary brain tumors are far more common than are primary brain tumors.

Any cancer can spread to the brain, but the most common types include:

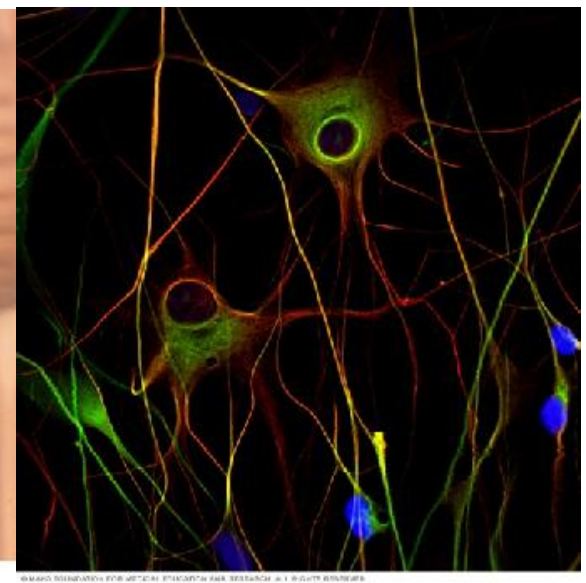

Breast cancer, Colon, Kidney, lung, Melanoma

Risk factors

\section{Probable Risk factors are:--}

\section{Gender}

In general, brain tumors are slightly more likely to occur in men than in women. Some specific types of brain tumors, such as Meningiomas, are more common in women.

Age

Most brain tumors in adults occur between the ages of $65-79$. Brain tumors also tend to occur in children younger than age 8. In children, malignant brain tumors are the leading cause of death from solid tumors (non-blood cell cancers).

\section{Ethnicity}

The risk for primary brain tumors in Caucasians is higher, as much as two-fold with Gliomas, than in people of other races. 


\section{Environmental or Occupational Risk Factors}

Exposure to ionizing radiation, usually from radiation therapy, is the only environmental risk factor that has been definitively linked to brain tumors. People who receive radiation therapy to the head during cancer treatment have an increased risk of developing brain tumors 10 - 15 years later. Workers in the nuclear industry are also at increased risk.

Despite much research, there is no evidence that electromagnetic field exposure from power lines or household appliances poses any risk. Several recent epidemiological studies have examined the cancer risk of wireless devices such as cellular (mobile) phones, which emit radiofrequency (RF) energy. To date, the studies do not show an association between cell phone use and common brain tumors like gliomas and Meningiomas. However, more research is needed to examine potential long-term effects, particularly for children and adolescents.

Researchers have also investigated a number of metals and chemicals including vinyl chloride, petroleum products, lead, arsenic, mercury, and pesticides. To date, there has been no clear evidence that implicates any specific industrial chemical or metal. Research continues.

\section{Medical Conditions}

People with impaired immune systems have an increased risk of developing central nervous system lymphomas. Organ transplantation, HIV infection, and chemotherapy are some medical factors that can weaken the immune system.

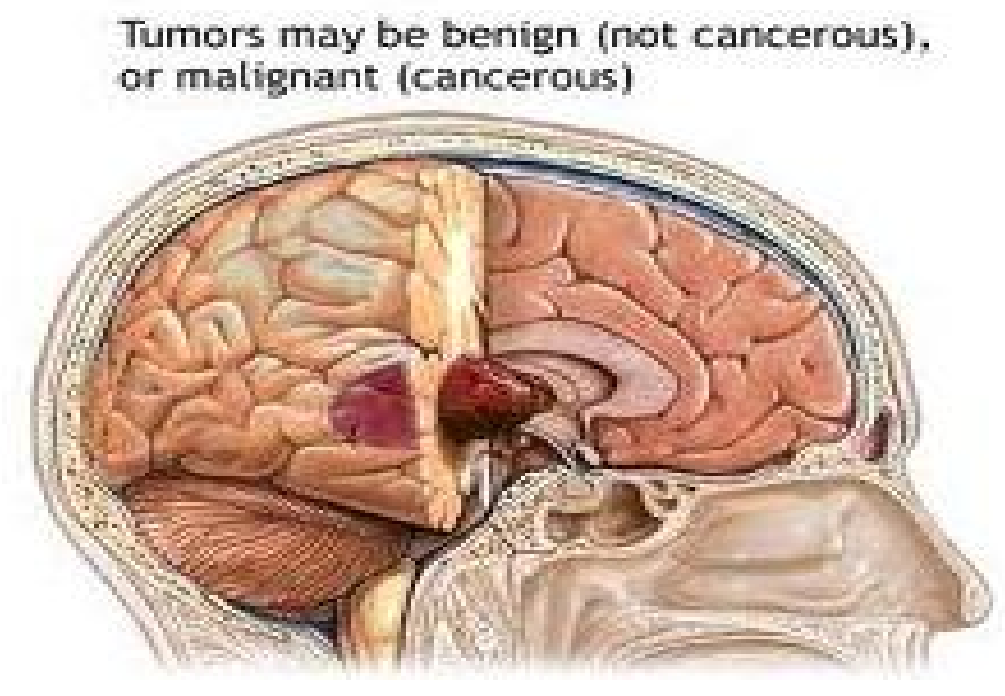

\section{Diagnosis}

\section{Diagnosis of brain tumors}

- Magnetic resonance imaging (MRI) is used to scan your brain and nerve tissues.

- A cranial computed tomography (CT) scan is equally useful.

- An electroencephalogram

(EEG) examines brain's electrical impulses.

- $\quad$ Tumor tissue biopsy may be examined to diagnose the tumor type.

- Cerebral spinal fluid can be tested for cancer cells.

\section{Conventional Radiotherapy}

Conventional radiotherapy uses external beams aimed directly at the tumor and is usually recommended for large or infiltrating tumors. It begins about a week after surgery and continues on an outpatient basis 5 days per week for 6 weeks. Older adults tend to have a more limited response to external-beam radiation therapy than younger people. Conventional external-beam radiation 
Int. J. Curr. Res. Med. Sci. (2017). 3(12): 13-21

\section{Location of Different Types of Brain Tumors}

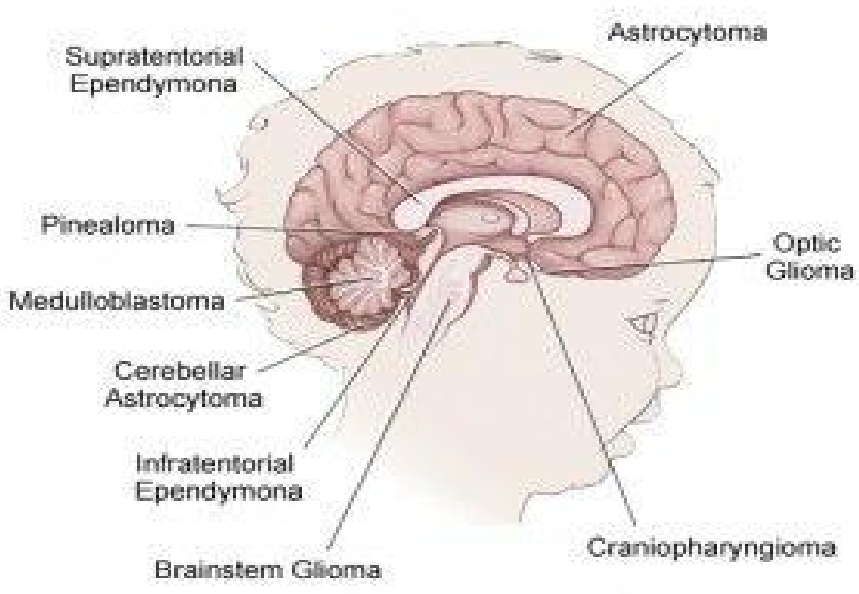

Exposure to radiation. People who have been exposed to a type of radiation called ionizing radiation have an increased risk of brain tumor. Examples of ionizing radiation include radiation therapy used to treat cancer and radiation exposure caused by atomic bombs.

More common forms of radiation, such as electromagnetic fields from power lines and radiofrequency radiation from cellphones and microwave ovens, have not been proved to be linked to brain tumors.

Family history of brain tumors. A small portion of brain tumors occur in people with a family history of brain tumors or a family history of genetic syndromes that increase the risk of brain tumors.

Primary brain tumors are much less common than are secondary brain tumors, in which cancer begins elsewhere and spreads to the brain.

Many different types of primary brain tumors exist. Each gets its name from the type of cells involved. Examples include:

Gliomas. These tumors begin in the brain or spinal cord and include astrocytoma, ependymomas, Glioblastomas, oligoastrocytomas and Oligodendroglioma.
Meningiomas. Meningiomas are a tumor that arises from the membranes that surround your brain and spinal cord (meninges). Most Meningiomas are noncancerous.

Acoustic neuroma (schwannoma). These are benign tumors that develop on the nerves that control balance and hearing leading from your inner ear to your brain.

Pituitary adenomas. These are mostly benign tumors that develop in the pituitary gland at the base of the brain. These tumors can affect the pituitary hormones with effects throughout the body.

Medulloblastomas. These are the most common cancerous brain tumors in children. A medulloblastomas starts in the lower back part of the brain and tends to spread through the spinal fluid. These tumors are less common in adults, but they do occur.

PNETs. Primitive neuroectodermal tumors (PNETs) are rare, cancerous tumors that start in embryonic (fetal) cells in the brain. They can occur anywhere in the brain.

Germ cell tumors. Germ cell tumors may develop during childhood where the testicles or ovaries will form. But sometimes germ cell tumors move to other parts of the body, such as the brain. 
Craniopharyngioma. These rare, noncancerous tumors start near the brain's pituitary gland, which secretes hormones that control many body functions. As the craniopharyngioma slowly grows, it can affect the pituitary gland and other structures near the brain.

\section{Cancer that begins elsewhere and spreads to the brain}

Secondary (metastatic) brain tumors are tumors that result from cancer that starts elsewhere in your body and then spreads (metastasizes) to brain. Secondary brain tumors most often occur in people who have a history of cancer. But in rare cases, a metastatic brain tumor may be the first sign of cancer that began elsewhere in your body. Secondary brain tumors are far more common than are primary brain tumors. Any cancer can spread to the brain, but the most common types include: In some patients with primary brain tumors, the cause of the tumor is not clear. But some factors do exist:--

In most people that may increase risk of a brain tumor. Risk factors include:

Age. Certain types of brain tumors occur almost exclusively in children or old age.

Exposure to radiation. People who have been exposed to a type of radiation called ionizing radiation have an increased risk of brain tumor. e.g. radiation therapy \& exposure caused by atomic explosion.

Family history of brain tumors. A small portion of brain tumors occur in people with a family history of brain tumors or a family history of genetic syndromes that increase the risk of brain tumors.

A neurological exam. A neurological exam may include, among other things, checking your vision, hearing, balance, coordination, strength and reflexes. Difficulty in one or more areas may provide clues about the part of your brain that could be affected by a brain tumor.
Imaging tests. Magnetic resonance imaging (MRI) is commonly used to help diagnose brain tumors. In some cases a dye may be injected through a vein in your arm during your MRI study.

A number of specialized MRI scan components - including functional MRI, perfusion MRI and magnetic resonance spectroscopy - may help your doctor evaluate the tumor and plan treatment.

Other imaging tests may include computerized tomography (CT) scan and positron emission tomography (PET).

Tests to find cancer in other parts of your body. If it's suspected that your brain tumor may be a result from another area of your body, doctor may recommend tests and procedures to determine where the cancer originated. One example might be a CT scan of the chest to look for signs of lung cancer.

Collecting and testing a sample of abnormal tissue (biopsy). A biopsy can be performed as part of an operation to remove the brain tumor, or a biopsy can be performed using a needle. A stereotactic needle biopsy may be done for brain tumors in hard to reach areas or very sensitive areas within your brain that might be damaged by a more extensive operation. Your neurosurgeon drills a small hole into your skull. A thin needle is then inserted through the hole. Tissue is removed using the needle, which is frequently guided by CT or MRI scanning.

The biopsy sample is then viewed under a microscope to determine if it is cancerous or benign. This information is critical to establish a diagnosis and prognosis and, most importantly, in guiding treatment.

\section{Treatment}

Treatment for a brain tumor depends on the type, size and location of the tumor, as well as your overall health and your preferences. 
Int. J. Curr. Res. Med. Sci. (2017). 3(12): 13-21

\section{Surgery}

If the brain tumor is located in a place that makes it accessible for an operation, your surgeon will work to remove as much of the brain tumor as possible.

In some cases, tumors are small and easy to separate from surrounding brain tissue, which makes complete surgical removal possible. In other cases, tumors can't be separated from surrounding tissue or they're located near sensitive areas in your brain, making surgery risky. In these situations your doctor removes as much of the tumor as is safe.

Even removing a portion of the brain tumor may help reduce your signs and symptoms.Surgery to remove a brain tumor carries risks, such as infection and bleeding. Other risks may depend on the part of your brain where your tumor is located. For instance, surgery on a tumor near nerves that connect to your eyes may carry a risk of vision loss.

\section{Radiation therapy}

Radiation therapy uses high-energy beams, such as X-rays or protons, to kill tumor cells. Radiation therapy can come from a machine outside your body (external beam radiation), or, in very rare cases, radiation can be placed inside your body close to your brain tumor (brachytherapy).

External beam radiation can focus just on the area of your brain where the tumor is located, or it can be applied to your entire brain (whole-brain radiation). Whole-brain radiation is most often used to treat cancer that has spread to the brain from some other part of the body.

Side effects of radiation therapy depend on the type and dose of radiation you receive. Common side effects during or immediately following radiation include fatigue, headaches and scalp irritation.

\section{Radiosurgery}

\section{Gamma Knife targeting, Stereotactic Radiosurgery}

Stereotactic Radiosurgery is not a form of surgery in the traditional sense. Instead, Radiosurgery uses multiple beams of radiation to give a highly focused form of radiation treatment to kill the tumor cells in a very small area. Each beam of radiation isn't particularly powerful, but the point where all the beams meet - at the brain tumor receives a very large dose of radiation to kill the tumor cells. There are different types of technology used in Radiosurgery to deliver radiation to treat brain tumors, such as a Gamma Knife or linear accelerator (LINAC).

\section{Chemotherapy}

Chemotherapy uses drugs to kill tumor cells. Chemotherapy drugs can be taken orally in pill form or injected into a vein (intravenously). The chemotherapy drug used most often to treat brain tumors is temozolomide (Temodar), which is taken as a pill. Many other chemotherapy drugs are available and may be used depending on the type of cancer. Chemotherapy side effects depend on the type and dose of drugs received.. Chemotherapy can cause nausea, vomiting and hair loss.

\section{Targeted drug therapy}

Targeted drug treatments focus on specific abnormalities present within cancer cells. By blocking these abnormalities, targeted drug treatments can cause cancer cells to die.

One targeted drug therapy used to treat a type of brain cancer called glioblastoma is bevacizumab (Avastin). This drug, given through a vein (intravenously), stops the formation of new blood vessels, cutting off blood supply to a tumor and killing the tumor cells. The targeted drug everolimus (Afinitor) is used to treat a benign brain tumor that occurs in people with a genetic disorder called tuberous sclerosis. Everolimus 
blocks an enzyme in the body that plays a role in the growth of cancer cells.

\section{Treatment of Complications}

\section{Peritumoral Edema}

Some tumors, particularly medulloblastomas, interfere with the flow of cerebrospinal fluid and cause hydrocephalus (accumulation of fluid in the skull), which in turn causes a build-up fluid in the ventricles (the cavities) in the brain. Symptoms of peritumoral edema include nausea and vomiting, severe headaches, lethargy, difficulty staying awake, seizures, visual impairment, irritability, and tiredness.

Corticosteroids (commonly called steroids), such as dexamethasone (Decadron), are used to treat peritumoral edema. Side effects include high blood pressure, mood swings, and increased risk of infection, stronger appetite, facial swelling, and fluid retention.

\section{Seizures}

Seizures are common in brain tumor cases, with younger patients having higher risks than older ones. Anti-seizure (anti-convulsant) medications, such as carbamazepine or phenobarbital, may treat seizures and are helpful in preventing recurrence. These drugs are not useful in preventing a first seizure, however, and they should not be used routinely to treat patients with newly diagnosed brain tumors. Anti-seizure medications should be used only for patients who have experienced a seizure.

Anti-seizure medications can interact with some of the chemotherapies used to treat brain cancers, including paclitaxel, irinotecan, interferon, and retinoic acid. Patients should discuss these interactions with their doctors.

\section{Depression}

Antidepressants can help treat the emotional side effects of associated with brain tumors. Support groups can also have great benefit for both patients and families.

\section{Prognosis}

Recent advances in surgical and radiation treatments have significantly extended average survival times of patients with brain tumors. These new treatments can often help reduce the size and progression of malignant Gliomas.

\section{References}

1.Bowers DC, Liu Y, Leisenring W, McNeil E, Stovall M, Gurney JG, et al. Late-occurring stroke among long-term survivors of childhood leukemia and brain tumors: a report from the Childhood Cancer Survivor Study. J Clin Oncol. 2006 Nov 20;24(33):5277-82. Epub 2006 Nov 6.

2.Buckner JC, Brown PD, O'Neill BP, Meyer FB, Wetmore CJ, Uhm JH. Central nervous system tumors. Mayo Clin Proc. 2007 Oct;82(10):127186.

3.Clarke J, Butowski N, Chang S. Recent advances in therapy for glioblastoma. Arch Neurol. 2010 Mar;67(3):279-83.

4. Frei P, Poulsen AH, Johansen C, Olsen JH, Steding-Jessen M, Schüz J. Use of mobile phones and risk of brain tumours: update of Danish cohort study. BMJ. 2011 Oct 19;343:d6387. doi: 10.1136/bmj.d6387.

5.Gerstner ER, Batchelor TT. Primary central nervous system lymphoma. Arch Neurol. 2010 Mar;67(3):291-7.

6.Iwamoto FM, Fine HA. Bevacizumab for malignant gliomas. Arch Neurol. 2010 Mar;67(3):285-8.

7.Linskey ME, Andrews DW, Asher AL, Burri SH, Kondziolka D, Robinson PD, et al. The role of stereotactic radiosurgery in the management of patients with newly diagnosed brain metastases: a systematic review and evidence-based clinical practice guideline. J Neurooncol. 2010; 96:45-68. Epub 2009 Dec. 4.

8. Mitchell DA, Sampson JH. Toward effective immunotherapy for the treatment of malignant brain tumors. Neurotherapeutics. 2009 Jul;6(3): 527-38. 
9.Nathan PC, Patel SK, Dilley K, Goldsby R, Harvey J, Jacobsen C, et al. Guidelines for identification of, advocacy for, and intervention in neurocognitive problems in survivors of childhood cancer: a report from the Children's Oncology Group. Arch Pediatr Adolesc Med. 2007 Aug; 161(8):798-806.

\begin{tabular}{|l|l|}
\hline \multicolumn{2}{|c|}{ Access this Article in Online } \\
\hline & Website: \\
\hline & www.ijcrims.com \\
\hline Quick Response Code & Subject: \\
& Medical \\
\hline
\end{tabular}

How to cite this article:

Anil Batta. (2017). Increasing Incidence of Brain Tumors. Int. J. Curr. Res. Med. Sci. 3(12): 13-21. DOI: http://dx.doi.org/10.22192/ijcrms.2017.03.12.003 